\title{
Stochastic Single Footfall Trace Model for Pedestrian Walking Load
}

\author{
Jun Chen ${ }^{* a, b}$, Guo Ding ${ }^{\mathrm{a}}$, Stana Živanovićc \\ ${ }^{a}$ Department of Structural Engineering, Tongji University, 1239 Si Ping Rd., Tumu Building, \\ Room A409, 200092, Shanghai, P. R. China \\ b State Key Laboratory of Disaster Reduction in Civil Engineering, Tongji University, \\ Shanghai, P. R. China \\ ${ }^{c}$ School of Engineering, University of Warwick, Coventry, CV4 7AL, UK \\ *Corresponding author
}

\begin{abstract}
Developing a model for the dynamic force generated by a pedestrian's foot on a supporting structure (single footfall trace model) is crucial to advanced numerical analysis and vibration serviceability assessment of the structure. A reliable model needs to re $^{\circ}$ ect the inter-subject and intra-subject randomness of human walking. This paper introduces a stochastic single footfall trace model in the form of a Fourier series in which body weight, walking frequency, and the first eight harmonics are treated as random variables. An experiment used 73 test subjects, walking at a range of pacing frequencies, to record force time histories and the corresponding gait parameters. Two variability descriptors were used to indicate inter-subject and intrasubject randomness. Further statistical analysis identi- ed the relationships between key parameters as well as the probability distribution functions of random variables. In the final step, an application of the proposed single footfall trace model was developed and tested. The proposed model represented the experimental data well in both time and frequency domains.
\end{abstract}

Keywords: Single footfall trace (SFT); vibration serviceability; walking; inter-subject variability; intra-subject variability. 


\section{Introduction}

Modern slender structures such as footbridges, long-span floors and staircases can be sensitive to walking-induced vibrations because of their low natural frequencies and low structural damping ratios. In turn, excessive structural vibrations can cause the occupants sitting in or standing on the structure to perceive uncomfortable or even intolerable conditions [1-4]. In rare cases, excessive vibrations can lead to safety problems when structures are damaged to the point of collapsing [5]. Walking-induced vibrations can also impair the function of vibration sensitive equipment in hospitals, high-tech factories and laboratories. With the rapid development of high strength and light weight construction materials, structures are becoming lighter, longer and taller than ever before. Examples in China include a pre-stressed concrete floor in a sports stadium with a span of $42 \mathrm{~m}$ [6] a beam-free composite floor in a residential building designed to be $10.0 \mathrm{~m}$ long and $9.8 \mathrm{~m}$ wide [7] and the recently completed Zhangjiajie Grand Canyon Glass Footbridge, which measures 430m long and 6m wide [8]. Vibration serviceability was identified as the key design consideration in these buildings because rectifying this issue in a completed structure is difficult and often cost-prohibitive. Thus, reliable evaluation of the vibration performance of flexible structures at the design stage is crucial in preventing failures in the future.

A reliable load model is a prerequisite for predicting human-induced structural vibrations. Given that walking is the most common activity within a structure, extensive experimental studies have been conducted on walking loads and several numerical models have been proposed and reviewed in literature [9,10]. A large number of experimental records of walking loads are necessary to establish a reliable mathematical model. Force plates are commonly used to record walking loads in experiments, but because of their relatively small size, they can usually accommodate only a single footfall trace (SFT). However, 2-4 force plates are often used to record successive SFTs, fromwhich a continuous force time history can be derived. Although a treadmill integrated with force plates is sometimes adopted to measure continuous walking load curves $[11,12]$, this device is prohibitively expensive for many research establishments and predefined treadmill belt speeds might reduce the variability in test subject's gait parameters. Moreover, disciplines like biomechanics [13] and sports science [14] traditionally use force plates for collecting SFT records. As a result, a large proportion of the current walking load databases around the world consist of SFTs. 
Mathematical representations of walking loads can be broadly divided into two types: deterministic and stochastic. The deterministic models usually assume that the walking load is a periodic time series in which force is identical in each step. Consequently, a Fourier series is used to model the loading function. Such models were incorporated in the design guidelines in the UK [15], USA [16], Canada [17] and Japan [18]. They differ from each other in the number of forcing harmonics considered in the series (generally from 1 to 4 ) and the corresponding parameters (Fourier coefficients and phase angles). The drawback of deterministic models is that they neglect the randomness in human walking, i.e. the fact that no two successive steps can be identical. In fact, human walking load is a kind of stochastic process by nature, it has inter-subject variability, e.g. different pacing rate, velocity, step length, load amplitude and body weight among humans, and intra-subject variability, e.g. the inability of a person to repeat the same force in each step [19]. Neglecting these variabilities in the load model will lead to overestimation of structural responses as pointed out by Brownjohn et al. [11] and Ding and Chen [20]. This is because randomness in real walking produces leakage of load energy around each harmonic center frequency, rather than creating a perfect concentration of energy at each harmonic in the deterministic load model. Stochastic load models order a better alternative. A stochastic walking load model suggested by Živanović et al. [19] treats some parameters in the Fourier series model as random variables, including walking frequency, step length and amplitude of walking force. As a further improvement, Racic and Brownjohn [12] proposed a data-driven stochastic walking load model which relies on random parameters being drawn from an experimental database, resulting in an exceptionally detailed representation of both temporal and spectral features of the walking-generated force.

Interestingly, in contrast to the large amount of work published on models for continuous walking load time history as mentioned above, there is a paucity of articles addressing the SFT model. A mathematical model for SFT has the following potential applications: (1) to fully use the database of walking load records, from various disciplines such as biomechanics [13] and sport science [14] to study randomness and avoid the erroneous assumption that all footfalls are identical; (2) to serve as a base function for reconstructing crowd loads when the time instant and spatial positions of heel strike and toe off events for each foot are available from measurements such as video and inertia sensor technologies [21,22]; (3) for advanced analysis of structures such as staircases on which individual steps are applied on different structural components, and (4) to facilitate finite element software development for structural vibration serviceability analysis, where it is possible to account for the spatial position of each heel strike. 
With these applications in mind, this paper aims to develop a stochastic model for a SFT in the vertical direction for walking on flat surfaces. The same framework can be used for developing SFT models for walking on stairs. The conceptual framework of the proposed model is introduced in Sec. 2. Using experimental records, the probabilistic features of the model's variables are analyzed in Sec. 3. The application and validation of the suggested model follows in Sec. 4. Finally, the main findings of this study are summarized in Sec. 5.

\section{Stochastic Model}

This section describes the framework for development of the stochastic model for SFT. It includes definitions of the parameters featured in the model as well as the parameters used to describe the variability in the generated force.

\subsection{Single footfall trace}

While walking, a person's body accelerates and decelerates intermittently resulting in a threedimensional dynamic force. This force is applied to the supporting surface at discrete points where each foot touches the ground. Only the largest, vertical component of the force $\mathrm{Fz}(\mathrm{t})$ is considered in this study. Typical records of two successive SFTs (solid lines), as well as their sum (dashed line), normalized by the test subject's body weight are shown in Fig. 1. The gray shaded area in the same figure indicates a double support phase of a walking cycle (when both feet are in contact with the ground).

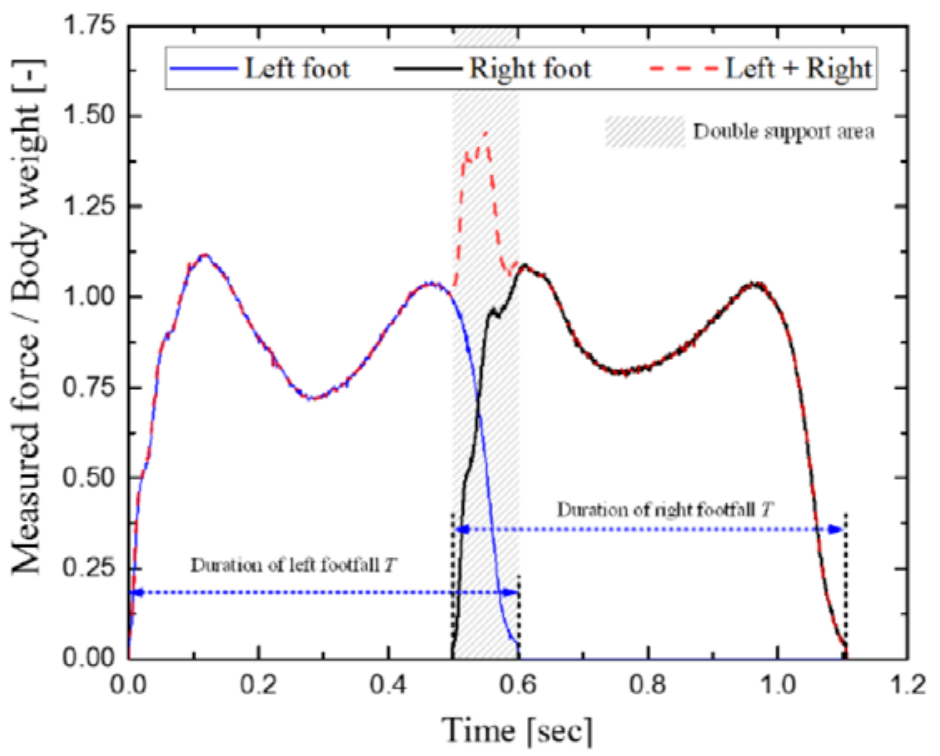

Fig. 1. A record of two consecutive footfalls (male, $85 \mathrm{~kg}$, pacing rate $2.0 \mathrm{~Hz}$ ). 


\subsection{Stochastic modeling of the single footfall trace}

A single footfall dynamic force $\mathrm{Fz}(\mathrm{t})$ can be expressed via a Fourier series function, as follows:

$$
F_{z}(t)=G\left[a_{0}+\sum_{k=1}^{n} a_{k} \sin \left(\frac{2 \pi k}{T} t+\varphi_{k}\right)\right],
$$

where $\mathrm{G}$ is the pedestrian's body weight, $\mathrm{a}_{0}$ is the mean value of the weight normalized force, $\mathrm{a}_{\mathrm{k}}$ and $\varphi_{\mathrm{k}}$ are the amplitude and phase angle of the kth component in the series (hereafter, kth harmonic), $\mathrm{T}$ is the duration of a single footfall (Fig. 1) and $\mathrm{n}$ is the number of harmonics to be considered. Furthermore, ak represents the dynamic load factor (DLF) of a SFT (referred hereafter as the DLF only) and it therefore should not be confused with the DLF of the total dynamic force, typically reported in literature discussing structural engineering applications.

In the vibration serviceability assessment of pedestrian structures, frequency of walking $f_{p}$ is accepted as a key random parameter, and it is usually expressed in $\mathrm{Hz}$ (i.e. the number of steps per second). For this reason, the frequency of walking in this study is treated as a primary random variable, while other random variables $\left(T, a_{0}, a_{k}\right.$ and $\left.\varphi_{k}\right)$ in Eq. (1) are tested for correlation with $\mathrm{f}_{\mathrm{p}}$. The remaining variable $\mathrm{G}$ in Eq. (1) is another independent random variable [23]. Therefore, the stochastic model of SFT in Eq. (1) has $2 n+4$ random variables, $f_{p}, G, a 0, T$, $\mathrm{a}_{\mathrm{k}}$ and $\varphi_{\mathrm{k}}$, where $\mathrm{k}=1 ; 2 ; \ldots ; \mathrm{n}$. Characteristics of these variables will be discussed in Sec. 3.

\subsection{Inter- and intra-subject variability}

As mentioned above, the randomness in the SFT originates from two sources: intersubject variability and intra-subject variability. The former denotes differences in SFTs between different persons in a population of walkers, The latter refers to differences in SFTs generated by the same person [10].

All variables in Eq. (1) possess both types of variability, apart from G which is characterized by inter-subject variability only. Let $\mathrm{x}$ represent a variable possessing both types of variability. The inter-subject variability descriptor for variable $\mathrm{x}$ can then be defined as

$$
\xi_{x}^{i}=\frac{\bar{x}_{i}}{\bar{x}},
$$

where $\bar{x}_{i}$ is the mean value of the parameter (over a series of steps) for the ith person, and $\bar{x}$ is the mean value of parameter $\mathrm{x}$ on the observed population of pedestrians, i.e. $\bar{x}$ is the population mean of all $\bar{x}_{i}$. The inter-subject descriptor, therefore, quantifies how much each person's mean differs from the mean of the population. 
The intra-subject variability descriptor of variable $\mathrm{x}$, on the other hand, can be

$$
\zeta_{x}^{i}(j)=\frac{x^{i}(j)}{\bar{x}_{i}}
$$

where $x^{i}(j)$ is the value of the parameter $\mathrm{x}$ in the jth step for the ith person. The intra-subject descriptor quantifies how much each step for the ith person differs from the mean value of the parameter for the same person.

Based on the two descriptors, parameter $\mathrm{x}$ for the ith person in his/her jth walking step can be expressed as

$$
x^{i}(j)=\xi_{x}^{i} \cdot \zeta_{x}^{i}(j) \cdot \bar{x} .
$$

Using this expression in practical applications requires determining population mean $\bar{x}$ and the probability distributions of $\xi_{x}^{i}$ and $\zeta_{x}^{i}(j)$. Specifics about these two distributions can be obtained from experimental data.

\section{Probability Distribution Functions of Model Parameters}

This section describes an experimental program designed for identifying the model parameters. It investigates potential correlations between different parameters and determines relevant probability distributions.

\subsection{Experimental programme}

The motion capture system Vicon and two force plates (type: AMTI OR6-7) were flushmounted to the laboratory floor to record the kinematics and kinetics of 73 volunteers while walking. The dimensions of the force plate were $464 \mathrm{~mm} \times 508 \mathrm{~mm} \times 82.55 \mathrm{~mm}$. There were 59 males (mean age plus standard deviation: $23.4 \pm 2.3$ yrs, weight: $65.1 \pm 4.1 \mathrm{~kg}$; height: $1.71 \pm$ $0.074 \mathrm{~m}$ ) and 14 females (age: $22.8 \pm 1.25 \mathrm{yrs}$, weight: $51.2 \pm 8.8 \mathrm{~kg}$; height: $1.62 \pm 0.025 \mathrm{~m}$ ) that took part in the experiments in a gait lab in Shanghai. There is evidence that no clinical difference in ground reaction forces exists between young and old subjects [22]. The participants were students or staff of Tongji University. Selection of the test participants and the test protocol satisfied the requirements of the Medical Ethics Committee of Tongji University.

Thirty-nine reflective markers were firmly attached to the anatomical landmarks of each participant's body (Fig. 2). Each test subject was then instructed to step on the two force plates in two successive steps, practicing several times so that they would step fully on the force plate 
by natural gait during the real test. Apart from the force plates recording the SFTs for the two footsteps, 10 infrared cameras in the Vicon system recorded spatial trajectories of the markers. The data collected for the three markers on each foot was used only for identifying the duration of SFTs, the step length and for eliminating suspicious experimental records, e.g. when one's foot did not fully land on the force plate. The sampling frequency of the force plate was set as $1000 \mathrm{~Hz}$. A detailed description of the walking experiments can be found elsewhere [25].

Each volunteer completed seven test cases: three free-walking cases (self-selected slow, normal and fast walking) and four metronome-controlled walking cases (at 1.5, 1.75, 2.0 and $2.25 \mathrm{~Hz}$ ). Each case consisted of the test subject producing either 9 or 10 SFTs in total. The program yielded 4814 SFTs of good quality. Each SFT was accompanied by the corresponding gait parameters, which were determined by Vicon system including the duration of the SFT, the achieved walking frequency (which was not necessarily the same as the target frequency in the metronome-controlled tests), the step length and the duration of the double-support phase. The test subject's body weight was used to normalize each SFT Eq. (1) and was then used to fit the normalized SFT curve to determine the corresponding model parameters, i.e. a $0, a_{k}$ and $\varphi_{\mathrm{k}}$. Finally, the experimental records were analyzed statistically to quantify the parameters required for defining the SFT model.

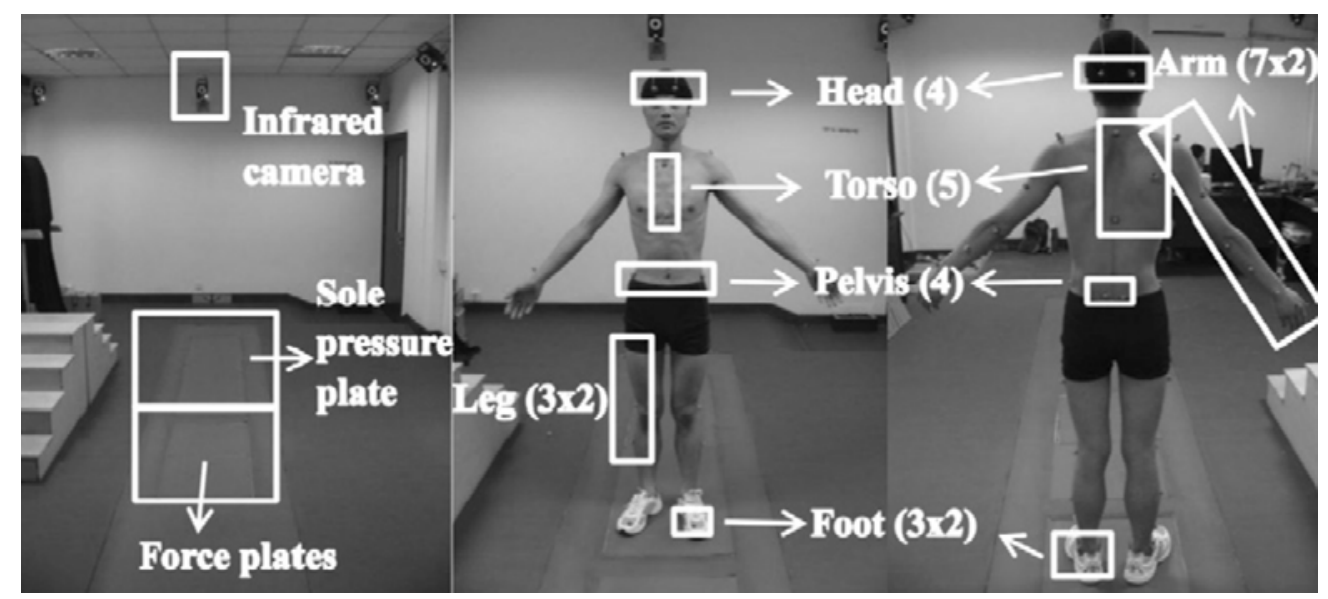

Fig. 2. Test subject with reflective markers participating in the experiment.

\section{2. $T$ and $a_{0}$ as functions of the walking frequency}

Figures 3 and 4 show how $\mathrm{T}$ and $\mathrm{a}_{0}$ each correlate with the achieved walking frequency $\mathrm{f}_{\mathrm{p}}$, respectively. All the SFTs records are used to plot Figs. 3 and 4. In both cases, the data scatter is relatively low and $\mathrm{T}$ and $\mathrm{a}_{0}$ both are functions of $\mathrm{f}_{\mathrm{p}}$, indicating that they are not independent 
variables. Regression analysis was used to establish the correlations with $f_{p}$ (solid lines in the two figures) as follows:

$$
\begin{gathered}
T=0.195 f_{p}^{2}-1.118 f_{p}+2.056, \\
a_{0}=0.031 f_{p}+0.75 .
\end{gathered}
$$

Equations (5) and (6) calculate the duration $\mathrm{T}$ (in seconds) and coefficient a0 (dimensionless number) for a given walking frequency $\mathrm{f}_{\mathrm{p}}(\mathrm{in} \mathrm{Hz}$ ) at each step. The value of coefficient a increases very slightly with walking frequency and is around 0.8 since it is related to a SFT only. For the analysis of a total force, this coefficient would be equal to 1.0, which shows that the mean value of the total dynamic force is equal to the weight of the walker.

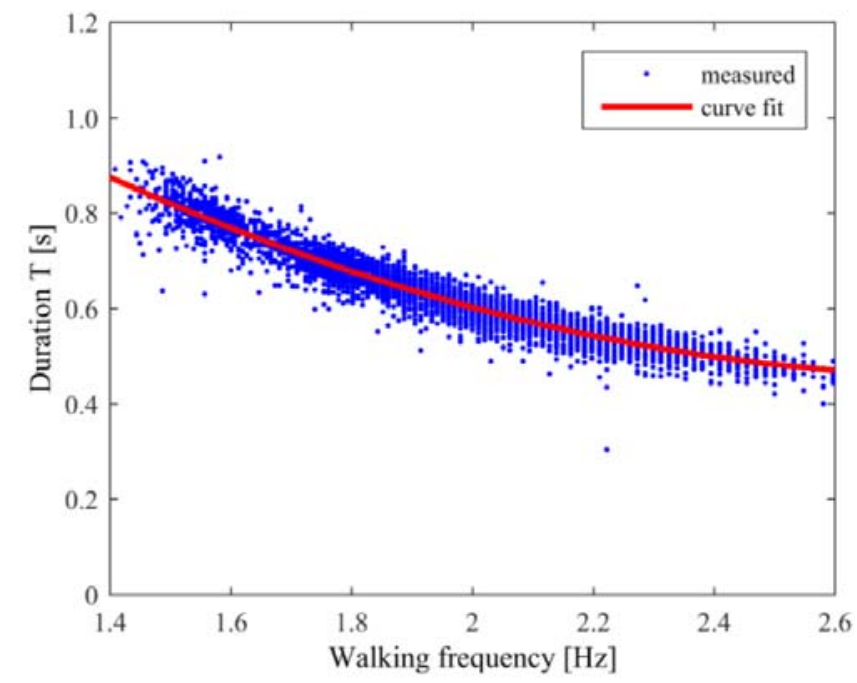

Fig. 3. Relationship between duration of the single footfall and walking frequency.

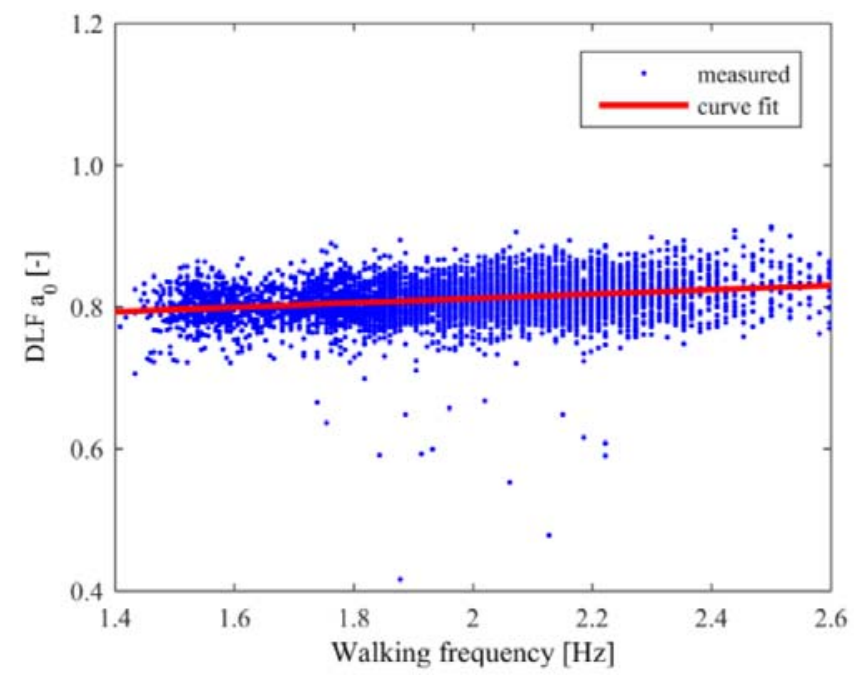

Fig. 4. Relationship between the constant coe \pm cient of SFT and walking frequency. 


\subsection{Population mean for $a_{k}$ and $\varphi_{\mathrm{k}}$ as functions of the walking frequency}

By trial and error, it was established that $n=8$ harmonics are required for a successful reconstruction of SFT in the time domain (Fig. 5). Namely, it was found that using fewer than eight harmonics might result in a continuous force time history that fails to describe the double support area sufficiently.

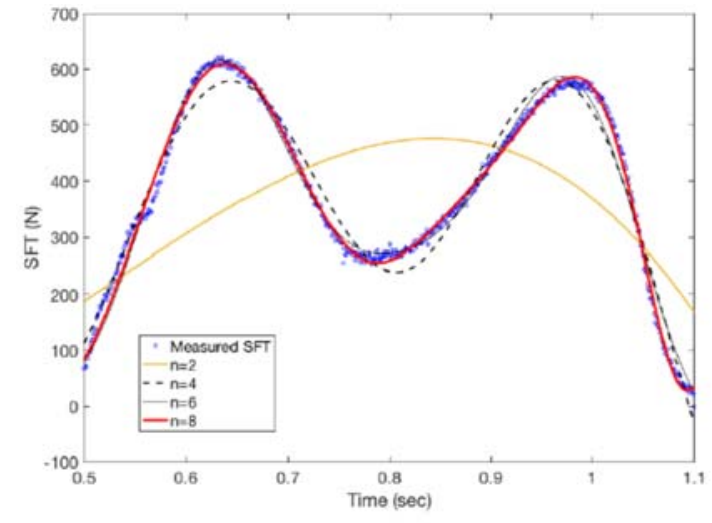

(a) SFT of left foot

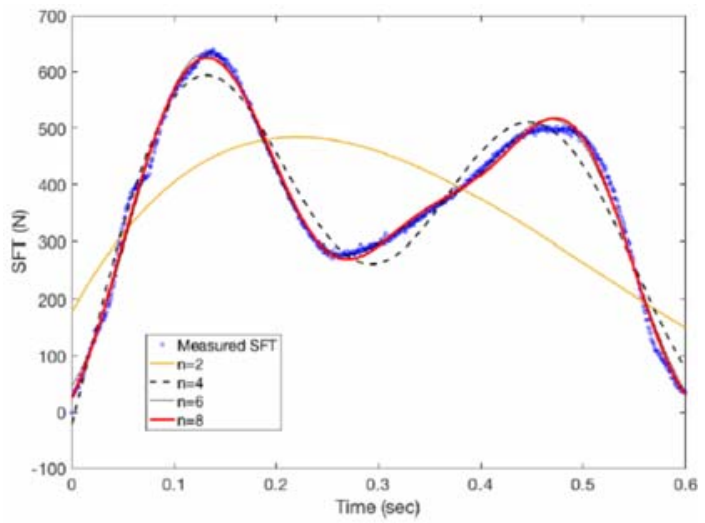

(b) SFT of right foot

Fig. 5. Comparison of measured SFT with its representation by $n=2,4,6$ and 8 harmonics (test walking frequency: $2.0 \mathrm{~Hz})$.

Figures 6(a)-6(h) show the first eight DLFs as functions of the walking frequency for all measured SFTs. The results for the corresponding phase angles are shown in Figs. 7(a)-7(h). The first four DLFs and phase angles exhibit some correlation with the walking frequency, while the higher harmonics seem to be random. Large scatter in these figures demonstrate that both inter-variability and intra-variability need to be considered for these variables. Assuming all 16 variables have a conditional mean value at a given walking frequency $f_{p}$ [26] regression analysis was performed to determine the relationship of the mean values and $f_{p}$. The resulting mathematical expressions are summarized in Table 1 and their plots are shown in Figs. 6 and 7 by solid lines.

Table 1. Mathematical expressions for conditional mean values of DLFs and phase angles at given walking frequency $\mathbf{f}_{\mathrm{p}}$.

\begin{tabular}{lcc}
\hline Order & Dynamic load factor & Phase angle (rad) \\
\hline 1 & $0.2080 f_{p}^{2}-0.9373 f_{p}+1.1998$ & $0.1730 f_{p}^{2}-0.4087 f_{p}-0.2936$ \\
2 & $0.1714 f_{p}+0.0025$ & $0296 f_{p}-0.5275$ \\
3 & $0.0129 f_{p}+0.1216$ & $0.0815 f_{p}-0.6305$ \\
4 & $0.0188 f_{p}+0.0235$ & $0.0955 f_{p}-0.5477$ \\
5 & 0.0364 & -0.3563 \\
6 & 0.0214 & -0.4004 \\
7 & 0.0146 & -0.3863 \\
8 & 0.0114 & -0.0851 \\
\hline
\end{tabular}




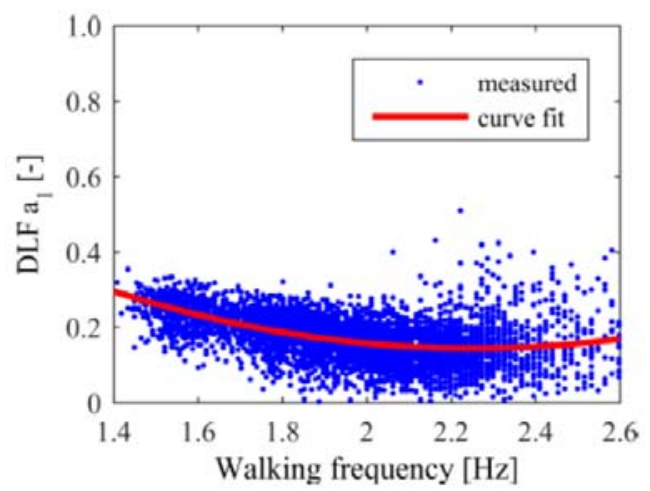

(a) DLF $a_{1}$

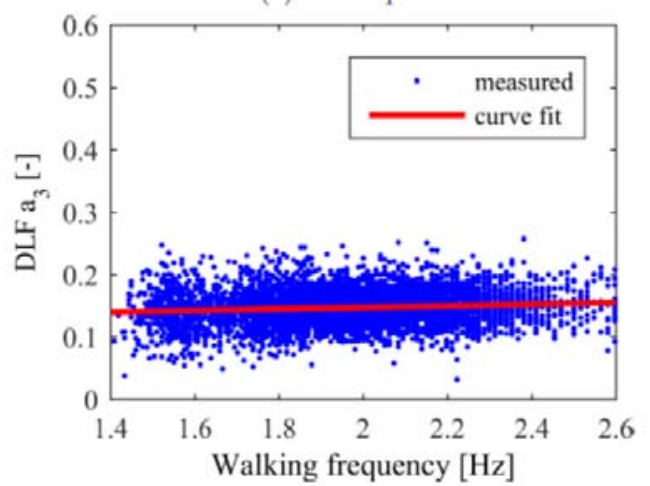

(c) DLF $a_{3}$

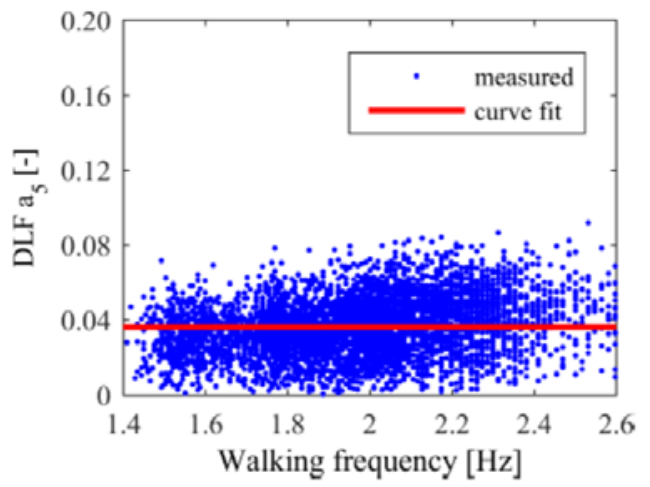

(e) DLF $a_{5}$

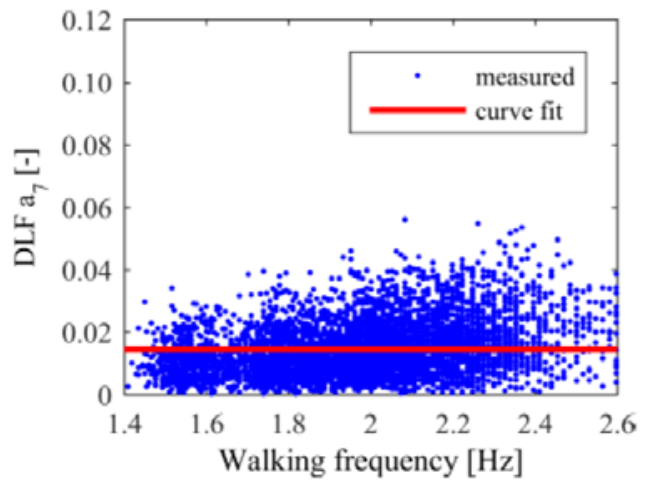

(g) DLF $a_{7}$

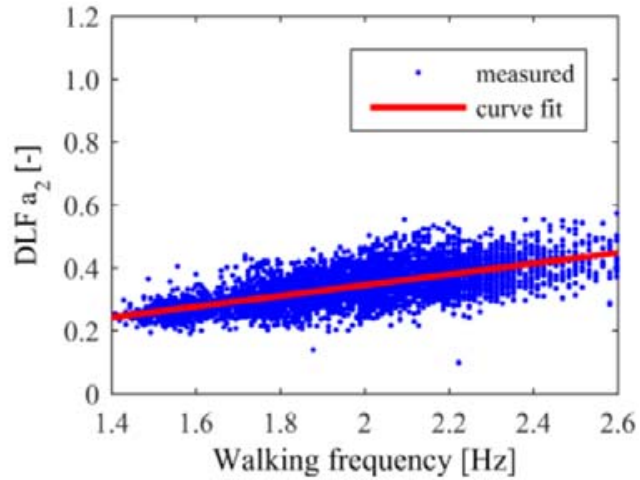

(b) DLF $a_{2}$

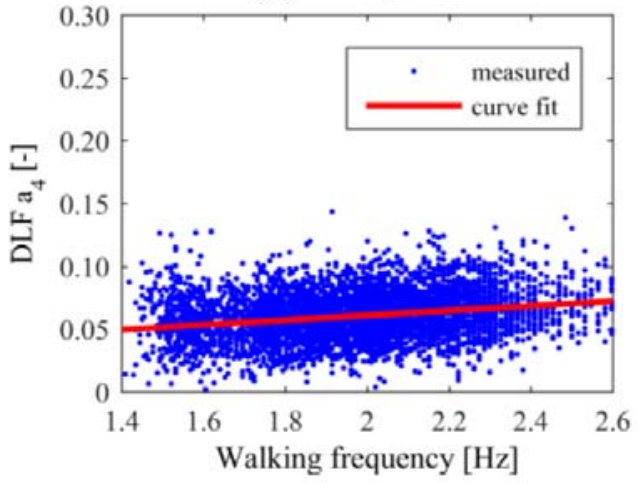

(d) DLF $a_{4}$

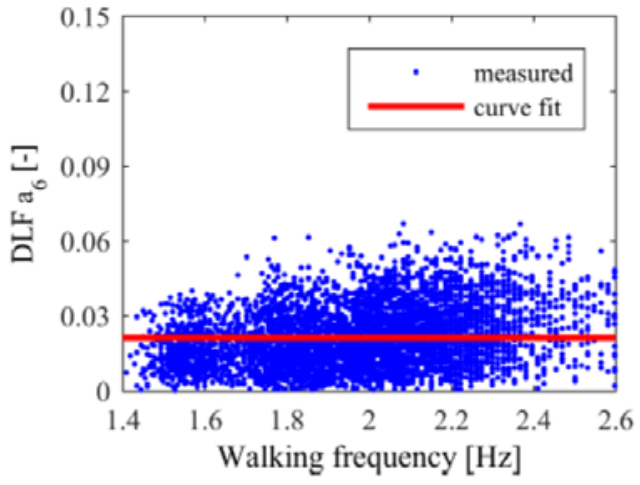

(f) DLF $a_{6}$

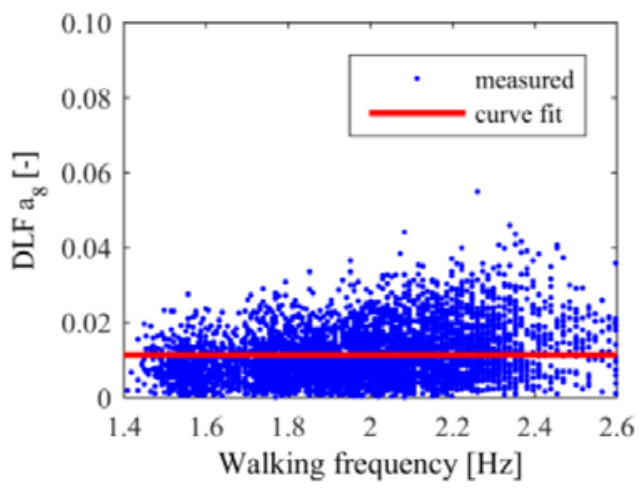

(h) DLF $a_{8}$

Fig. 6. Variation of DLFs with walking frequency. 


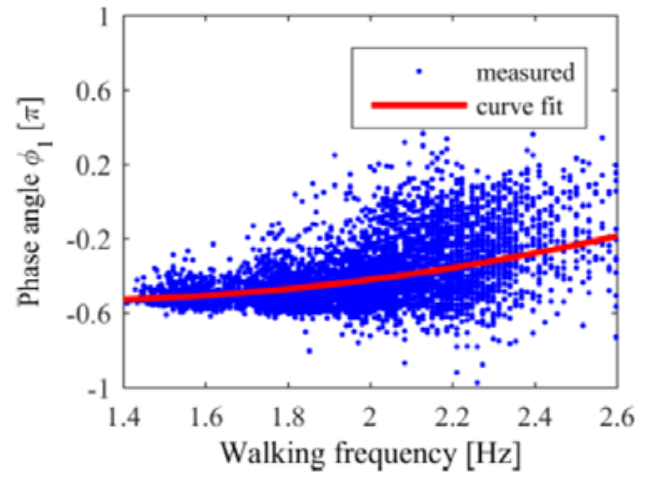

(a) Phase angle $\varphi_{1}$

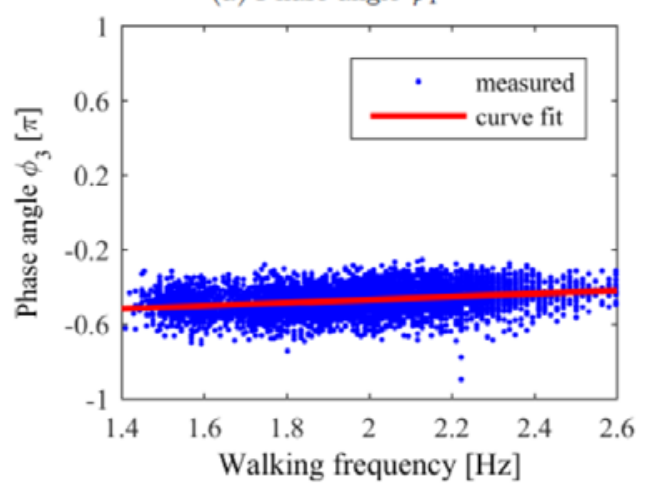

(c) Phase angle $\varphi_{3}$

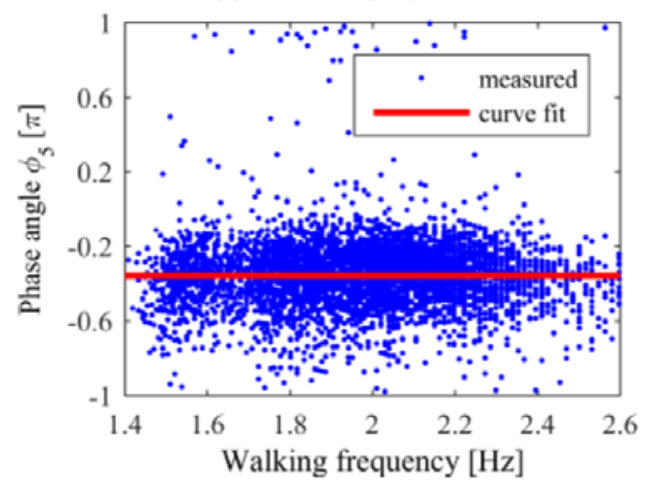

(e) Phase angle $\varphi_{5}$

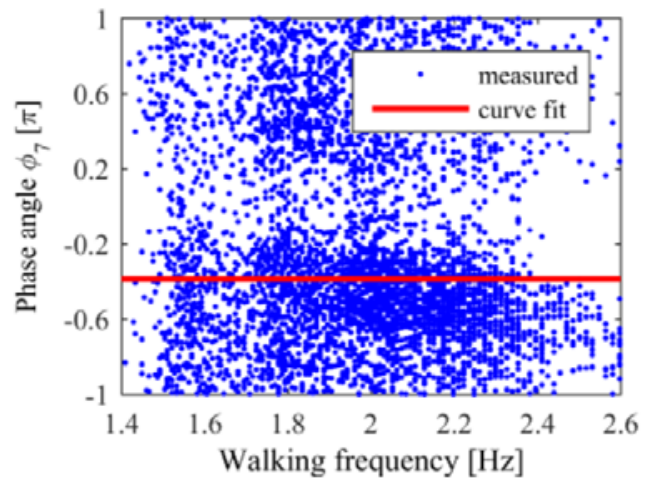

(g) Phase angle $\varphi_{7}$

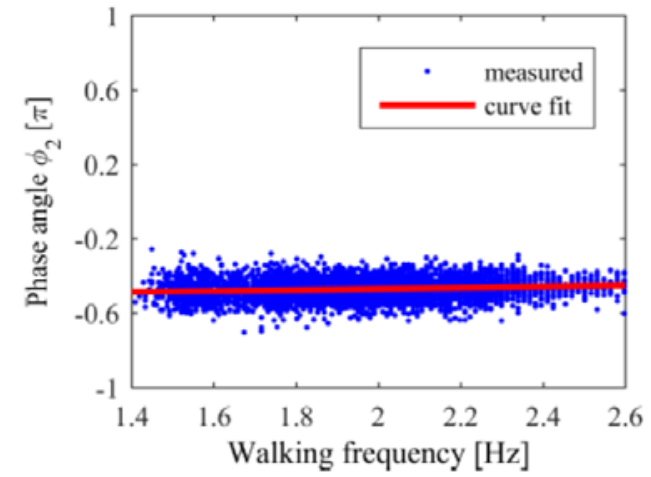

(b) Phase angle $\varphi_{2}$

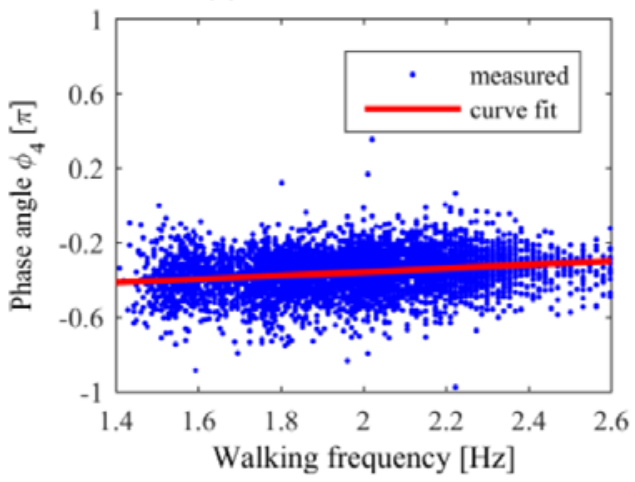

(d) Phase angle $\varphi_{4}$

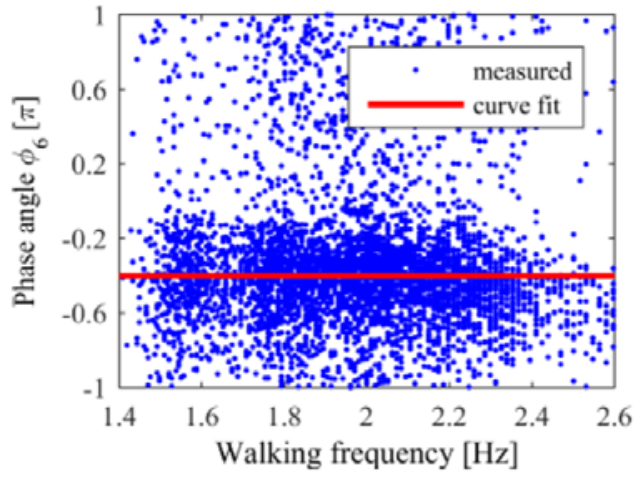

(f) Phase angle $\varphi_{6}$

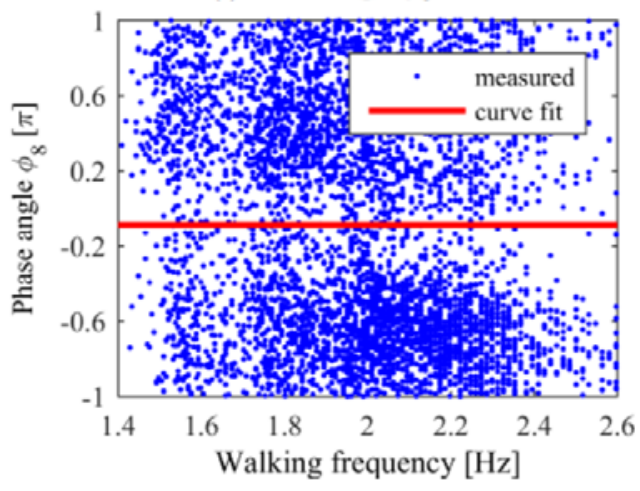

(h) Phase angle $\varphi_{8}$

Fig. 7. Variation of phase angles with walking frequency. 


\subsection{Inter-subject variability of $a_{k}$ and $\varphi_{k}$}

Equation (2) calculated the inter-subject variability coe \pm cient for the 16 variables ( $\mathrm{a}_{\mathrm{k}}$ and $\varphi_{\mathrm{k}}$ ) at each walking frequency. Statistical analysis of $\xi_{\mathrm{ak}}$ and $\xi_{\varphi \mathrm{k}}(\mathrm{k}=1-8)$ showed that they follow normal distributions $\mathrm{N}(1, \sigma)$. The results for $\xi_{\text {al }}$ and $\xi_{\varphi 1}$ are shown in Fig. 8. The standard deviations $\sigma$ for all variables were frequency dependent. The linear functions shown in Table 2 approximate these relationships.

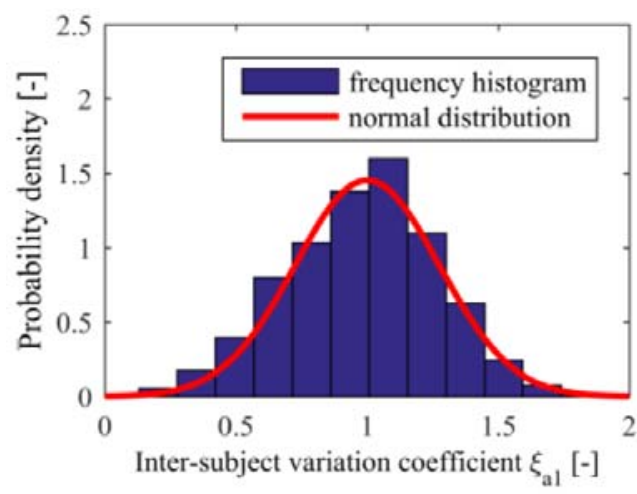

(a)

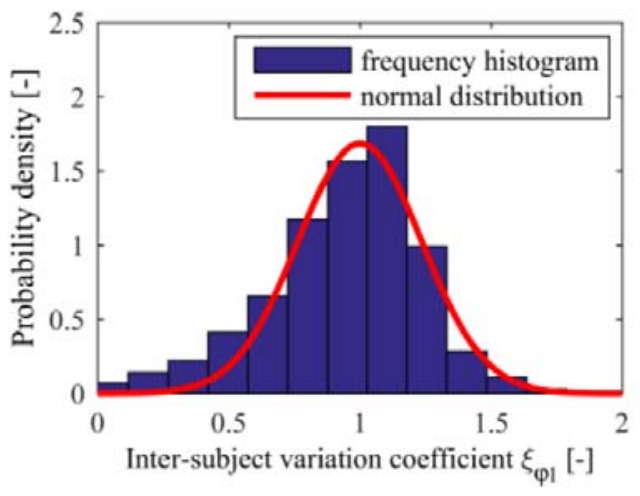

(b)

Fig. 8. Statistical analysis result of $\xi_{\mathrm{a} 1}$ and $\xi \varphi_{1}$.

Table 2. Standard deviation of inter-subject variability $\xi_{\mathrm{ak}}$ and $\xi \varphi_{\mathrm{k}}$ (normal distribution with mean value 1.0).

\begin{tabular}{lrl}
\hline Order & Dynamic load factor & \multicolumn{1}{c}{ Phase angle $(\mathrm{rad})$} \\
\hline 1 & $0.301 f_{p}-0.323$ & $0.1730 f_{p}^{2}-0.4087 f_{p}-0.2936$ \\
2 & $0.031 f_{p}+0.053$ & $0.0296 f_{p}-0.5275$ \\
3 & $-0.054 f_{p}+0.264$ & $0.037 f_{p}+0.054$ \\
4 & $-0.112 f_{p}+0.474$ & $0.002 f_{p}+0.244$ \\
5 & $0.116 f_{p}+0.116$ & $-0.060 f_{p}+0.436$ \\
6 & $0.244 f_{p}-0.015$ & $-0.021 f_{p}+0.428$ \\
7 & $0.352 f_{p}-0.224$ & $-0.214 f_{p}+0.880$ \\
8 & $0.362 f_{p}-0.252$ & $-1.323 f_{p}+5.735$ \\
\hline
\end{tabular}

The linear correlation coe \pm cients between the $16 \xi_{\text {ak }}$ and $\xi_{\varphi \mathrm{k}}$ coefficients are given in Table 3 . The coefficient matrix presented in Table 3 is symmetrical. The majority of the correlation coefficients have values lower than 0.4 implying a weak correlation. A few exceptions, underlined and bolded in the table, exist for DLFs for higher order harmonics as well as for the two correlation coe \pm cients involving the phase angles. The DLFs related to these exceptions, e.g. a4 - a7, have much smaller absolute amplitudes compared to those with weak correlations, e.g. a1 and a2, implying that they will have less influence in the final shape of a 
simulated SFT. These findings suggest, that for the purpose of load simulation, the 16 descriptors of inter-subject variability can be assumed to be independent random variables.

Table 3. Correlation coefficients of intra-subject variability $\xi_{\mathrm{ak}}$ and $\xi \varphi_{\mathrm{k}}$.

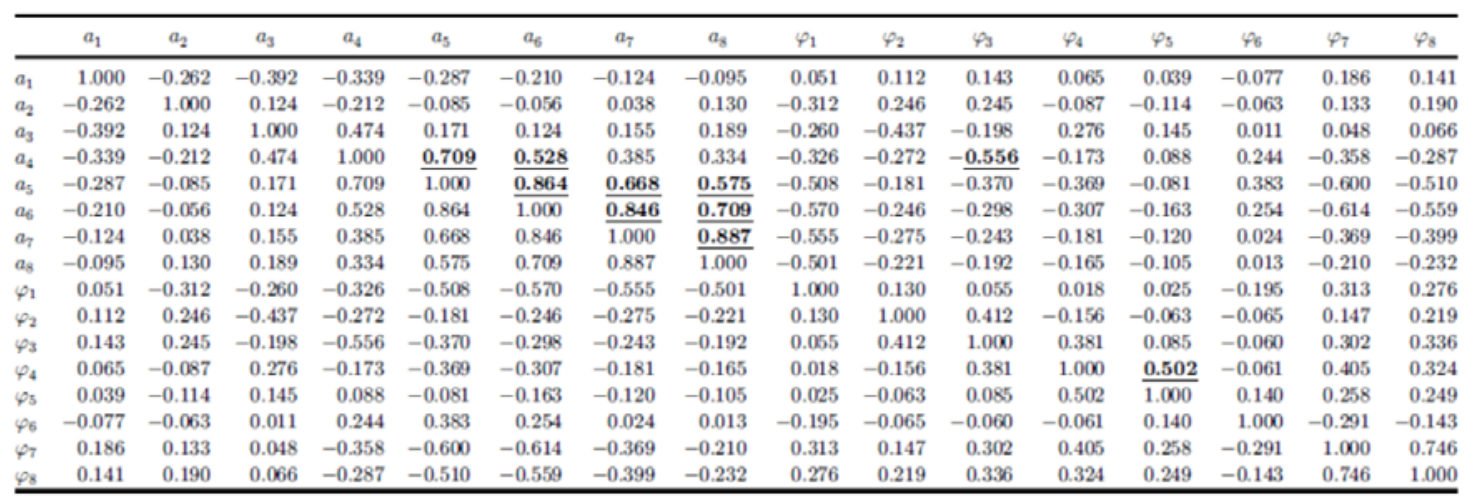

\subsection{Intra-subject variability of $a_{k}$ and $\varphi_{k}$}

The intra-subject variability $\zeta_{\mathrm{ak}}$ and $\zeta_{\varphi \mathrm{k}}$ for each of the 16 variables was calculated using Eq. (3). This is demonstrated in an example related to a1. Suppose a test subject produced nine SFTs in a test involving a single pacing frequency. The ratio of a1 for each SFT over the mean value of the nine SFTs is found and it represents the intra-subject variability. Records for all the test subjects from the same test frequency were statistically analyzed. For each test case, results demonstrated that the intra-subject variability follows a normal distribution and the standard deviation is nearly the same. That is to say, within the walking frequency range of 1.5-2.25 Hz considered in this study, the intra-subject variability of $\mathrm{a}_{\mathrm{k}}$ and $\varphi_{\mathrm{k}}$ is frequency independent. Therefore, data from all test subjects for all four walking frequencies were used to determine the normal distribution $\mathrm{N}(1, \sigma)$ for the intra subject variability descriptors. The results are summarized in Table 4.

Again, linear correlation coefficients among the 16 intra-subject variabilities were calculated and the results are given in Table 5. All except two correlation coefficients are less than 0.4, implying a weak correlation. Thus, we can treat the intra-subject variabilities of ak and $\varphi_{\mathrm{k}}$ as independent variables. 
Table 4. Standard deviation of intra-subject variability $\zeta_{\mathrm{ak}}$ and $\zeta_{\varphi \mathrm{k}}$ (normal distribution with mean value 1.0).

\begin{tabular}{lcc}
\hline Order & Dynamic load factors & Phase angles \\
\hline 1 & 0.123 & 0.102 \\
2 & 0.059 & 0.058 \\
3 & 0.101 & 0.074 \\
4 & 0.158 & 0.145 \\
5 & 0.202 & 0.209 \\
6 & 0.296 & 0.186 \\
7 & 0.363 & 0.219 \\
8 & 0.378 & 0.201 \\
\hline
\end{tabular}

Table 5. Correlation coe \pm cients of inter-subject variability $\zeta_{\mathrm{ak}}$ and $\zeta_{\varphi \mathrm{k}}$.

\begin{tabular}{|c|c|c|c|c|c|c|c|c|c|c|c|c|c|c|c|c|}
\hline & $a_{1}$ & $a_{2}$ & $a_{3}$ & $a_{4}$ & $a_{5}$ & $a_{6}$ & $a_{7}$ & $a_{8}$ & $\varphi_{1}$ & $\varphi_{2}$ & $\varphi_{3}$ & $\varphi_{4}$ & $\varphi_{5}$ & $\varphi_{6}$ & $\varphi_{7}$ & $\varphi_{8}$ \\
\hline$a_{1}$ & 1.000 & -0.187 & -0.303 & -0.150 & -0.227 & -0.231 & -0.162 & -0.128 & -0.062 & 0.279 & 0.184 & 0.061 & 0.001 & -0.005 & 0.141 & 0.134 \\
\hline$a_{2}$ & -0.187 & 1.000 & 0.262 & -0.207 & -0.170 & -0.142 & -0.009 & 0.016 & -0.010 & -0.229 & 0.024 & 0.060 & 0.008 & -0.016 & 0.173 & 0.174 \\
\hline$a_{3}$ & -0.303 & 0.262 & 1.000 & 0.313 & 0.032 & 0.000 & 0.040 & 0.060 & -0.024 & -0.424 & -0.220 & 0.333 & -0.017 & 0.011 & 0.131 & 0.097 \\
\hline$a_{4}$ & -0.150 & -0.207 & 0.313 & 1.000 & 0.526 & 0.236 & 0.069 & 0.083 & -0.021 & -0.084 & -0.389 & 0.043 & 0.006 & 0.034 & 0.002 & 0.085 \\
\hline$a_{5}$ & -0.227 & -0.170 & 0.032 & 0.526 & $\overline{1.000}$ & 0.483 & 0.133 & 0.095 & -0.012 & -0.082 & -0.316 & -0.241 & 0.016 & 0.033 & -0.257 & -0.077 \\
\hline$a_{6}$ & -0.231 & -0.142 & 0.000 & 0.236 & 0.483 & 1.000 & 0.442 & 0.260 & 0.014 & -0.086 & -0.232 & -0.223 & 0.038 & 0.020 & -0.347 & -0.213 \\
\hline$a_{7}$ & -0.162 & -0.009 & 0.040 & 0.069 & 0.133 & 0.442 & 1.000 & 0.652 & 0.020 & -0.152 & -0.159 & -0.083 & 0.020 & 0.003 & 0.008 & -0.088 \\
\hline$a_{8}$ & -0.128 & 0.016 & 0.060 & 0.083 & 0.095 & 0.260 & 0.652 & $\overline{1.000}$ & 0.029 & -0.138 & -0.144 & -0.052 & 0.017 & 0.024 & 0.105 & -0.006 \\
\hline$\varphi_{1}$ & -0.062 & -0.010 & -0.024 & -0.021 & -0.012 & 0.014 & 0.020 & 0.029 & 1.000 & 0.102 & -0.019 & -0.172 & 0.000 & 0.001 & -0.018 & -0.024 \\
\hline$\varphi_{2}$ & 0.279 & -0.229 & -0.424 & -0.084 & -0.082 & -0.086 & -0.152 & -0.138 & 0.102 & 1.000 & 0.393 & -0.141 & -0.003 & 0.019 & -0.065 & -0.048 \\
\hline$\varphi_{3}$ & 0.184 & 0.024 & -0.220 & -0.389 & -0.316 & -0.232 & -0.159 & -0.144 & -0.019 & 0.393 & 1.000 & 0.261 & 0.019 & 0.006 & 0.052 & 0.023 \\
\hline$\varphi_{4}$ & 0.061 & 0.060 & 0.333 & 0.043 & -0.241 & -0.223 & -0.083 & -0.052 & -0.172 & -0.141 & 0.261 & 1.000 & 0.010 & 0.009 & 0.223 & 0.090 \\
\hline$\varphi s$ & 0.001 & 0.008 & -0.017 & 0.006 & 0.016 & 0.038 & 0.020 & 0.017 & 0.000 & -0.003 & 0.019 & 0.010 & 1.000 & 0.000 & 0.001 & 0.063 \\
\hline$\varphi_{6}$ & -0.005 & -0.016 & 0.011 & 0.034 & 0.033 & 0.020 & 0.003 & 0.024 & 0.001 & 0.019 & 0.006 & 0.009 & 0.000 & 1.000 & -0.003 & 0.005 \\
\hline$\varphi_{7}$ & 0.141 & 0.173 & 0.131 & 0.002 & -0.257 & -0.347 & 0.008 & 0.105 & -0.018 & -0.065 & 0.052 & 0.223 & 0.001 & -0.003 & 1.000 & 0.484 \\
\hline$\varphi_{8}$ & 0.134 & 0.174 & 0.097 & 0.085 & -0.077 & -0.213 & -0.088 & -0.006 & -0.024 & -0.048 & 0.023 & 0.090 & 0.063 & 0.005 & 0.484 & 1.000 \\
\hline
\end{tabular}

\subsection{Walking frequency $f_{p}$ and body weight $G$}

To simulate load at the design stage, the mean walking frequency $\bar{f}_{p}^{i}$ of an individual pedestrian within a group can be selected to meet any particular design requirement, such as (1) walking at a predefined frequency (e.g. to simulate a loading scenario where a sound stimulus is present), and (2) free walking. The designer should determine the walking frequency (or its distribution properties) according to various factors such as type of structure, criteria in local design guidelines, characteristics of the local population of pedestrians and similar aspects. For instance, a walking frequency to generate resonant response can be assigned to a walking group to simulate a severe loading scenario. For free walking, there are many available statistical results for pacing rates, such as $\mathrm{N}(1.99 \mathrm{~Hz}, 0.17 \mathrm{~Hz})$ by Matsumoto [27] and N $(1.87 \mathrm{~Hz}, 0.19$ Hz) by Živanović [28] for people walking across footbridges. Therefore, the parameters of the normal distribution of the mean pacing rate can be selected either from the literature or from site-specific data relevant for the structure under analysis. 
Having determined $\bar{f}_{p}^{i}$, the walking frequency at each walking step can be obtained by introducing intra-subject variability $\zeta_{p}^{i}(j)$, that is

$$
f_{p}^{i}(j)=\zeta_{p}^{i}(j) \cdot \bar{f}_{p}^{i}
$$

To determine the intra-subject variability at specific walking frequencies, data collected during fixed walking frequency test cases were analyzed using Eq. (3). It was found that the distribution of $\zeta_{p}^{i}(j)$ is relatively frequency independent. Moreover, Fig. 9 shows the variation of $\zeta_{p}^{i}(j)$ for walking frequency in free walking cases. The linear correlation coe \pm cient between the two variables is 0.248 indicating a very low correlation. Therefore, all the data (from both the fixed frequency tests and free walking tests) were merged, and a normal distribution $\mathrm{N}$ (1.0, $0.0325)$ for $\zeta_{p}^{i}(j)$ identified, as shown in Fig. 10.

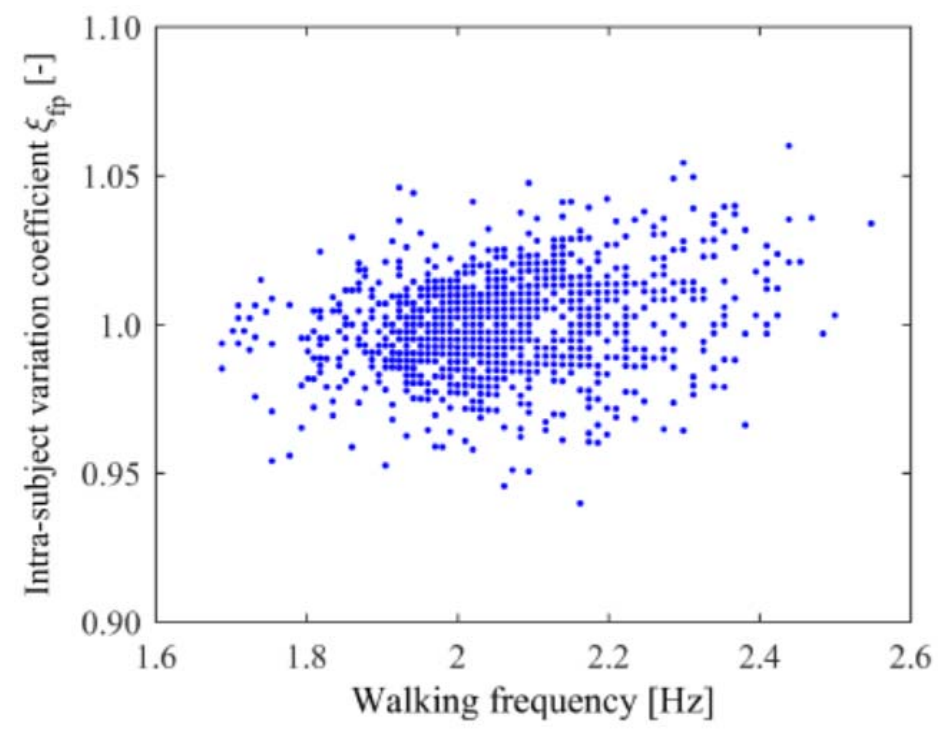

Fig. 9. Variation of $\zeta_{p}^{i}(j)$ of walking frequency for free walking case.

The parameter $\mathrm{G}$ in Eq. (1) is an independent variable characterized by only inter-subject variability. It can be randomly simulated based on its statistical properties. The body weight typically follows a normal distribution expressed as $\mathrm{N}(\mu, \sigma)$ or a log-normal distribution expressed as $\log \mathrm{N}(\mu, \sigma)$, where $\mu$ and $\sigma$ are mean value and standard deviation. Selection of values $\mu$ and $\sigma$ depend on factors such as race, gender, age, and living area (rural or urban). Xiao-guang et al. [29] collated statistics on a sample of 202,749 Chinese participants showing that for young adults (ages 18-44 yrs) and middle-age adults (ages 45-59 yrs) in urban areas, the body weight distributions were $\mathrm{N}(62.4 \mathrm{~kg}, 12.0 \mathrm{~kg})$ and $\mathrm{N}(64.5 \mathrm{~kg}, 10.9 \mathrm{~kg})$, respectively. 
For U.S. residents, Portier et al. [30] reported $\log \mathrm{N}(75.61 \mathrm{~kg}, 18.02 \mathrm{~kg})$ based on a survey of 13,462 adults aged $18-65$.

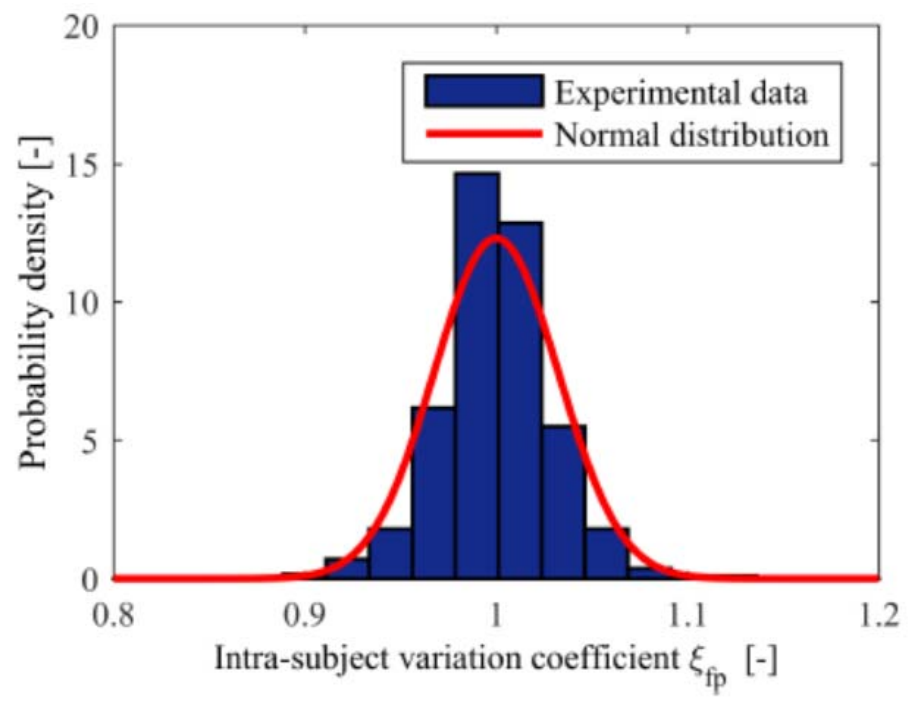

Fig. 10. Distribution of intra-subject variability for walking frequency.

For vibration serviceability design simulations, a pedestrian population can be modeled either by drawing body weight values from an appropriate distribution or by taking the mean value as representative for all pedestrians. The latter approach is especially justifiable for crowd simulations given that the vibration response is relatively insensitive to variations in average body weight [26]. In addition, body weight can also be selected as a percentile (other than 50\%) value, depending on the target value for the probability of non-exceedance of the vibration response.

\subsection{Final remarks on load simulations}

Two considerations remain for applying SFT model in the vibration serviceability assessment. The first is the spatial position of the pedestrian's first foot contact with the structure at the start of the crossing. The second is the duration of the double support phase during which the SFTs for each foot have to be overlapped. The position of the first contact point can be drawn from a uniform distribution $[0, \Delta \mathrm{L}]$, where the $\Delta \mathrm{L}$ is the average step length in the pedestrian population. The average value was $0.67 \mathrm{~m}$ in this study; but it will inevitably vary between different populations and different structures. The duration of the double support (DS) time can be determined on a step-by-step basis using the relationship DS(j)=T(j)-1/f $(j)$. 


\section{Verification}

\subsection{Application procedure for SFT simulation}

Based on the information provided so far by the suggested stochastic SFT model, the procedure for generating a time history for the ith person in a walking group can be divided into the following 10 steps:

1) Randomly generate a body weight $\mathrm{G}^{\mathrm{i}}$ for individual pedestrians by using the appropriate probability distribution function, or by assigning a representative percentile value.

2) Randomly generate the mean frequency of walking $\bar{f}_{p}^{i}$ for each pedestrian from an appropriate distribution by following suggestions in Sec. 3.6.

3) Randomly generate mean step length for each pedestrian from a frequency independent, normal distribution available in the literature. An example is $\mathrm{N}(0.74 \mathrm{~m}$, $0.08 \mathrm{~m}$ ) observed on a population of about 2000 people in Podgorica City in Montenegro [28]. Dividing the length of the path the pedestrian is walking by the mean step length results in the number of steps $M_{i}$ the ith pedestrian requires to cross the structure.

4) Determine the mean value of DLFs and phase angles for eight forcing harmonics using the equations given in Table 1 and $\bar{f}_{p}^{i}$ obtained in Step 2.

5) Randomly generate the inter-subject variability coefficient $\xi_{x}^{i}$ for DLFs and phase angles using normal distributions, whose parameters are available in Table 2.

6) For the jth step $\left(j=1, M_{i}\right)$, randomly generate the intra-subject variability coefficient $\zeta_{f p}^{i}(i)$ for walking frequency from $\mathrm{N}(1.0,0.0325)$. Calculate the walking frequency at the jth step using $f_{p}^{i}(j)=\zeta_{f p}^{i}(\mathrm{i}) \bar{f}_{p}^{i}$ then calculate $\mathrm{T}^{\mathrm{i}}(\mathrm{j})$ and $a_{o}^{i}(j)$ using Eqs. (5) and (6).

7) For the jth step, generate the intra-subject variability coefficients $\zeta_{x}^{i}(j)$ for the parameters of the forcing harmonics using the normal distribution parameters in Table 4. Calculate $a_{k}^{i}(\mathrm{j})$ and $\varphi_{k}^{i}(\mathrm{j}), \mathrm{k}=1-8$, at the jth step from Eq. (4), where $\bar{x}$, $\xi_{a k}^{i}$ and $\xi_{\varphi k}^{i}$ are obtained in Steps 3 and 4.

8) Introduce $G^{\mathrm{i}}, \mathrm{T}^{\mathrm{i}}(\mathrm{j}), a_{o}^{i}(\mathrm{j}), a_{k}^{i}(\mathrm{j})$ and $\varphi_{k}^{i}(\mathrm{j})$ into Eq. (1) to calculate the single footfall load at the jth step for the ith pedestrian.

9) Repeat steps 5-7 until the SFTs for all the M steps are simulated. 
10) If a continuous walking load curve has to be constructed, merge every pair of successive steps using the overlapping time calculated from $\operatorname{DS}(\mathrm{j})=\mathrm{T}^{\mathrm{i}}(\mathrm{j})-1 / f_{p}^{i}(\mathrm{j})$.

This procedure can be used to develop a software program for generating walking load time histories, which can then be integrated into a finite element model of a structure [31].

\subsection{Verification I: Simulating SFT time history}

Figure 11 compares simulated SFTs (dashed lines) with experimental measurements (solid lines) for four different walking frequencies. Figure 12 compares simulated continuous walking load time histories from the SFT model and the other four existing deterministic models, namely ISO-10137 [32], Blanchard [33], Allen and Murray [34] and Young et al. [35]. Figure 11 shows that the simulated SFTs are similar to the measured records where peak values and time instances occur, as well as step duration and force waveform. Due to its stochastic nature, it is not easy to judge the SFT model's performance using the single continuous load example given in Fig. 12. Nevertheless, the waveform of the simulated time history is compatible with those from existing models.

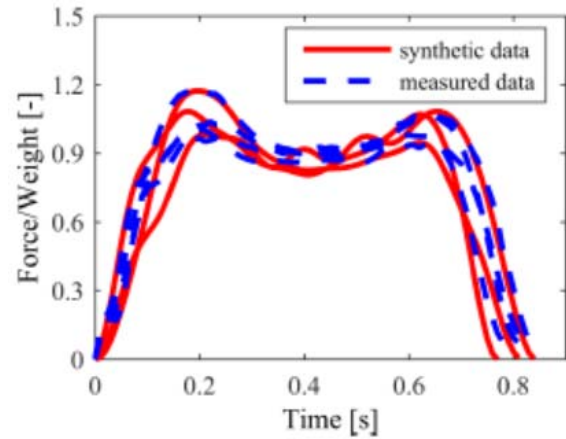

(a) $f_{\mathrm{p}}=1.5 \mathrm{~Hz}$

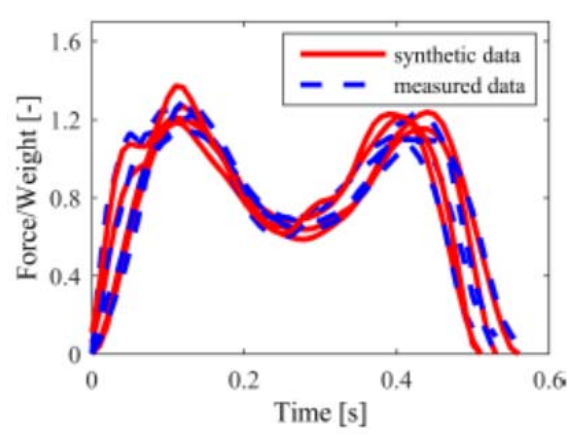

(c) $\vec{f}_{p}=2.0 \mathrm{~Hz}$

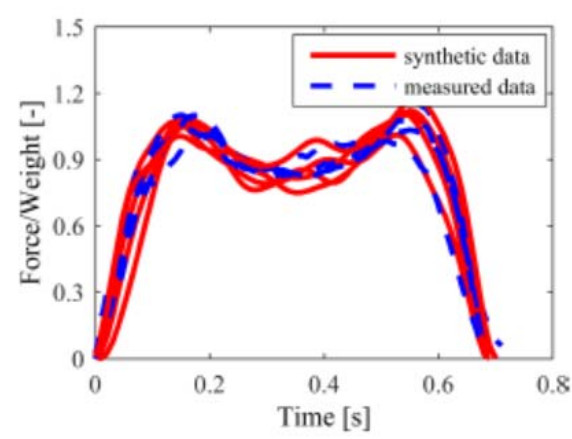

(b) $f_{p}=1.75 \mathrm{~Hz}$

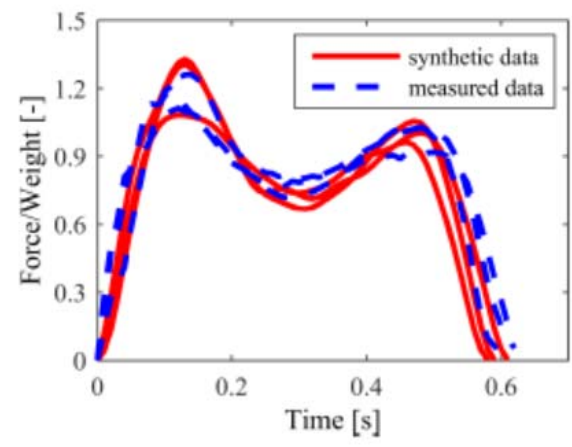

(d) $\hat{f}_{p}=2.25 \mathrm{~Hz}$

Fig. 11. Comparison between synthetic and measured SFTs. 


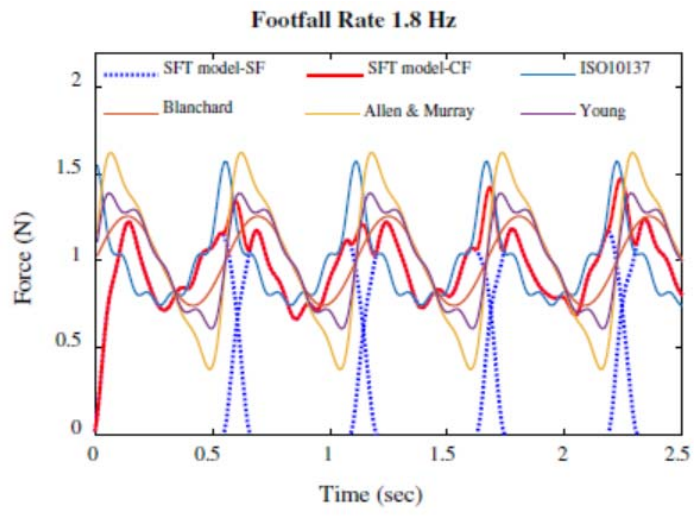

(a) $f_{p}=1.8 \mathrm{~Hz}$

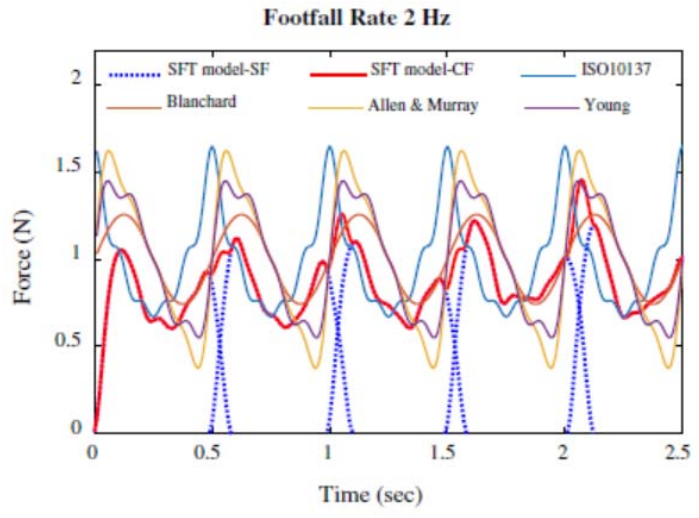

(b) $f_{p}=2.0 \mathrm{~Hz}$

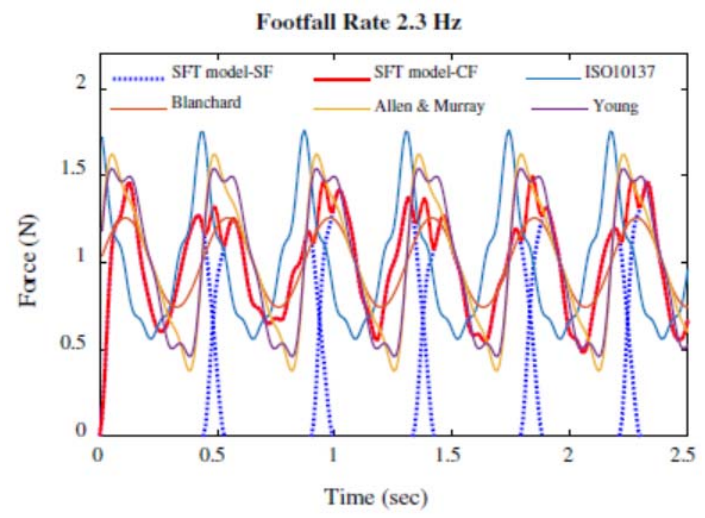

(c) $f_{p}=2.3 \mathrm{~Hz}$

Fig. 12. Comparations of simulated walking time histories from SFT and four different models.

\subsection{Verification II: structural response spectrum}

An important verification step in determining the performance of the model is to see if the model is capable of predicting a sufficiently accurate structural response for a range of structure to walking frequency ratios. To see how well the proposed model works for calculating vibration response, the simulated continuous load curves were applied individually to a singledegree-of-freedom (SDOF) structure system with unit mass. In the calculation, frequency range for SDOFs is 0.05 to $10 \mathrm{~Hz}$ with an interval of $0.05 \mathrm{~Hz}$ (i.e. in total $200 \mathrm{SDOFs}$ ), and 24,000 walking load time histories were simulated and applied to each SDOF (i.e. 24,000 x 200 times dynamic analysis). To be more specific, the walking loads were simulated for 100 people, with each person walking at 24 pacing rates, each of which was randomly selected in the range of 1.4-2.6 Hz. For every pacing rate, ten walking load curves were simulated and each curve had 62 SFTs, meaning in 24,000 x 62 SFTs were simulated by the proposed approach. The resulting acceleration response spectra from the above intensive calculations are shown in Fig. 13. Here, the simulated data is compared with the response spectrum that is calculated directly, i.e. by using the measured continuous force time histories collected by Chen et al. [36] It can be 
concluded that the spectrum obtained using simulated walking loads agrees well with that obtained using measured walking loads.

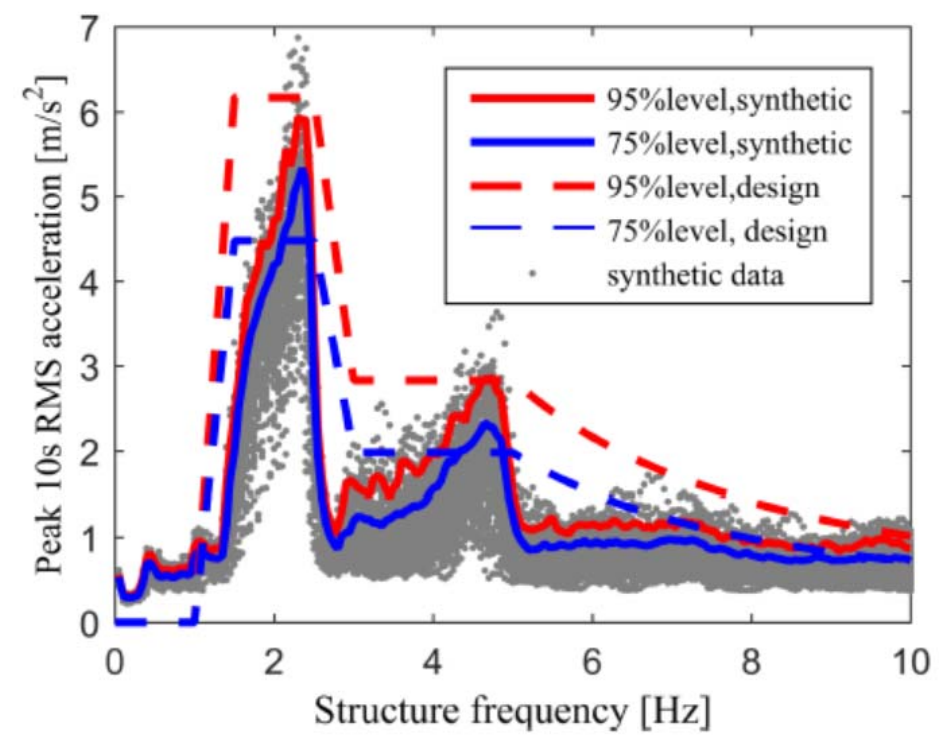

Fig. 13. Comparison between calculated SDOF response with response spectrum (damping ratio is 0.02$)$.

\section{Conclusions}

The study developed a stochastic mathematical model to represent single footfall trace for pedestrian walking. The model is defined as a Fourier series function in which body weight, pacing frequency and step length are independent random variables, while the trace duration, DLFs and phase angles for the first eight forcing harmonics are random variables that are functions of pacing frequency. The inter-subject and intra-subject randomness of pacing frequency and forcing harmonics were modeled by using two descriptors of variability. Experiments using 73 test subjects walking at a range of pacing frequencies resulted in a database of 4814 single footfall trace records. Statistical analysis of the experimental data was performed to establish the relationship between relevant parameters and derive relevant probability distribution functions. The procedure for generating single footfall load curves was then explained and the quality of its time and frequency domain representations verified. Finally, while the relationships among model parameters were determined using experimental data for 73 individuals drawn from an Asian population, the conceptual framework of the proposed model could readily be applied to other walking load databases to include a more diverse set of subjects, if and when required. 


\section{ACKNOWLEDGMENTS}

The authors would like to acknowledge the financial support provided by the National Natural Science Foundation of China (51778465, U1711264), State Key Laboratory for Disaster Reduction of Civil Engineering (SLDRCE14-B-16) as well by the UK Engineering and Physical Sciences Research Council [grant number EP/M021505/1: Characterizing dynamic performance of fiber reinforced polymer structures for resilience and sustainability]. Moreover, the authors would like to thank all the test subjects for participating in the project to make the data collection possible.

\section{References}

1. A. Ebrahimpour and R. L. Sack, A review of vibration serviceability criteria for floor structures, Comput. Struct. 83(28-30) (2005) 2488-2494.

2. C. M. R. Gaspar, J. G. Santos da Silva and L. F. Costa-Neves, Multimode vibration control of building steel-concrete composite ${ }^{\circ}$ oors submitted to human rhythmic activities, Comput. Struct. 165 (2016) 107-122.

3. X. H. Zhou, J. Li, J. P. Liu and Y. F. Chen, Dynamic performance charactristics of prestressed cable RC truss ${ }^{\circ}$ oor system under human-induced loads, Int. J. Struct. Stab. Dyn. 17(4) (2017).

4. R. J. Ma, L. Ke, D. L. Wang, A. R. Chen and Z. C. Pan, Experimental study on pedestrians' perception of human-induced vibrations of footbridges, Int. J. Struct. Stab. Dyn. 18(10) (2018).

5. X. H. Zhou, J. Li, J. P. Liu and Y. F. Chen, Dynamic performance charactristics of prestressed cable RC truss ${ }^{\circ}$ oor system under human-induced loads, Int. J. Struct. Stab. Dyn. 17(4) (2017).

6. B. Wolmuth and J. Surtees, Crowd-related failure of bridges, ICE — Civil Eng. 156(3) (2003) 115-123.

7. J. Chen, S. X. Yan and M. S. Zhang, Vibration performance assessment of a long-span concrete floor using field measurements over a five-year period, Adv. Struct. Eng. 17(8) (2014) $1145-1158$.

8. G. Ding, J. Chen, R. He and L. W. Ye, Vibration serviceability design and field tests of longspan ultra-slim composite floor system for super tall residential buildings, The 2016 World Congress on Advances in Civil, Environmental, and Materials Research, ICC Jeju, Jeju Island, Korea (2016).

9. Highestbridges, Zhangjiajie grand canyon glass footbridge, (2016) http://www.highestbridges.com/wiki/index.php?title=Zhangjiajie-Grand-Canyon-Glass-

Footbridge.

10. S. Živanović, A. Pavic and P. Reynolds, Vibration serviceability of footbridges under human-induced excitation: A literature review, J. Sound Vib. 279(1-2) (2005) 1-74.

11. V. Racic, A. Pavic and J. M. W. Brownjohn, Experimental identification and analytical modelling of human walking forces: Literature review, J. Sound Vibr. 326(1-2) (2009) 1-49. 
12. J. M. W. Brownjohn, A. Pavic and P. Omenzetter, A spectral density approach for modelling continuous vertical forces on pedestrian structures due to walking, Can. J. Civ. Eng. 31(1) (2004) 65-77.

13. V. Racic and J. M. W. Brownjohn, Stochastic model of near-periodic vertical loads due to humans walking, Adv. Eng. Inform. 25(2) (2011) 259-275.

14. R. Vera-Rodriguez et al., A large scale footstep database for biometric studies created using cross-biometrics for labelling, 2008 10th Int. Conf. Control Automation Robotics \& Vision: Icarv 2008, Vols. 1-4, Hanoi, Vietnam, pp. 1361-1366.

15. S. Khandelwal and N. Wickstrom, Evaluation of the performance of accelerometer-based gait event detection algorithms in different real-world scenarios using the MAREA gait database, Gait \& Posture 51 (2017) 84-90.

16. A. Pavic and M. R. Willford, Appendix G: Vibration serviceability of post-tensioned concrete floors, in Post-Tensioned Concrete Floor Design Handbook, 2nd edn. (Concrete Society, Slough, 2005), pp. 99-107.

17. T. M. Murrary, D. E. Allen and E. E. Ungar, Floor vibration due to human activity, American Institue of Steel Construction, Design Guideline \#11 (1997).

18. NRC, National Research Council of Canada, The Supplement to the National Building Code of Canda, Ottawa (1995).

19. AIJ, Architectural Institute of Japan, Recommendations for Loads on Buildings, Chap. 4, Japan (2004).

20. S. Živanović, A. Pavic and P. Reynolds, Probability-based prediction of multi-mode vibration response to walking excitation, Eng. Struct. 29(6) (2007) 942-954.

21. D. Guo and J. Chen, $\mathrm{In}^{\circ}$ uences of walking load randomness on vibration responses of longspan floors, J. Vibr. Eng. 29(1) (2016) 123-131.

22. Q. Li, J. Fan, J. Nie, Q. Li and Y. Chen, Crowd-induced random vibration of footbridge and vibration control using multiple tuned mass dampers, J. Sound Vib. 329 (2010) 4068-4092.

23. K. Van Nimmen, G. Lombaert, I. Jonkers, G. De Roeck and P. Van den Broeck, Characterisation of walking loads by 3D inertial motion tracking, J. Sound Vib. 333(20) (2014) 5212-5226.

24. F. Ricciardelli and A. David Pizzimenti, Lateral walking-induced forces on footbridges, J. Bridge Eng. ASCE 12(6) (2007) 677-688.

25. E. C. Jansen, D. Vittas, S. Hellberg and J. Hansen, Normal gait of young and old men and women: Ground reaction force measurement on a treadmill, Acta Orthop. Scand. 53(2) (1982) 1993-1996.

26. J. Chen, Y. Peng and Y. Ting, On methods for extending a single footfall trace into a continuous force curve for floor vibration serviceability analysis, Struct. Eng. Mech. 46(2) (2013) 179-196.

27. L. Pedersen and C. Frier, Sensitivity of footbridge vibrations to stochastic walking parameters, J. Sound Vib. 329(13) (2010) 2683-2701.

28. Y. Matsumoto et al., Dynamic design of footbridge, in Proc. IABSE (1978), P-17/78.

29. S. Živanović, Benchemark footbridge for vibraiton serviceability assessment under vertical component of pedestrian load, ASCE J. Struct. Eng. 138(10) (2012) 1193-1202.

30. Y. Xiao-Guang, L. Yan-ping, M. Guan-Sheng, H. Xiao-qi and W. Jing-zhong, Study on weight and height of the Chinese people and the di ${ }^{\circledR e r e n c e s}$ between 1992 and 2002, Chin. J. Epidemiol. 26(7) (2005) 489-493. (in Chinese) 
31. K. Portier, J. K. Tolson and S. M. Roberts, Body weight distributions for risk assessment, Risk Anal. 27(1) (2007) 11-26.

32. C. C. Caprani and E. Ahmadi, Formualtion of human-structure interaction system models for vertical vibration, J. Sound Vib. 377 (2016) 346-367.

33. ISO 10137, Base for Design of structure-serviceability of buildings and walkways against vibrations, Geneva.

34. J. Balnchard, B. L. Davies and J. W. Simth, Design criteria and analysis for dynamic loading of footbridges, Proc. Symp. Dynamic Behaviour of Birdges at the Transport and Road Research Laboratory, Berkshire, May, Soft Science 29(1) (1977) 77-87.

35. D. E. Allen and T. M. Murray, Design criterion for vibrations due to walking, AISC Eng. J. 30(4) (1993) 117-129.

36. M. Willford, P. Young and C. Field, Improved methodologies for the prediction of footfallinduced vibration, Proc. SPIE 5933, Buildings for Nanoscale Research and Beyond, California (2005).

37. J. Chen, R. T. Xu and M. S. Zhang, Acceleration response spectrum for predicting floor vibration due to occupant walking, J. Sound Vib. 333(15) (2014b) 3564-3579. 